\title{
Legitimación de la violencia en parejas heterosexuales desde el género como una de las estructuras de práctica social
}

\section{Legitimizers of violence in heterosexual couples, taking into account gender as a social practice structure}

\author{
Angélica Orozco Idárraga ${ }^{a}$ \\ (D) https://orcid.org/0000-0001-9990-0532 \\ E-mail: angelica.orozco®curnvirtual.edu.co \\ Aura Aguilar Carob \\ (D) https://orcid.org/0000-0002-7569-2422 \\ E-mail: auraguilarळmail.uniatlantico.edu.co \\ María Amarís Macías ${ }^{c}$ \\ (D) https://orcid.org/0000-0002-3229-7887 \\ E-mail: mamarisळuninorte.edu.co \\ ${ }^{a}$ Corporación Universitaria Rafael Núñez. Facultad de Ciencias \\ Sociales y Humanas. Departamento del Atlántico. Barranquilla, \\ Caribe, Colombia. \\ bUniversidad del Atlántico. Facultad de Educación. \\ Departamento del Atlántico. Barranquilla, Caribe, Colombia. \\ 'Universidad del Norte. Facultad de Humanidades y \\ Ciencias Sociales. Departamento del Atlántico. Barranquilla, \\ Caribe, Colombia.
}

\section{Correspondência}

Angélica Orozco Idárraga

Corporación Universitaria Rafael Núñez. Facultad de Ciencias

Sociales y Humanas. Carrera 54, n 66-54. Barranquilla, Colombia.

\section{Resumen}

Este artículo tuvo como objetivo develar las representaciones sociales de mujeres víctimas, agresoresyprestatarios/as de servicios legitimadoras de violencia de pareja desde la estructura de género y práctica social en el distrito de Barranquilla, Colombia. Esta es una investigación cualitativa, con diseño fenomenológico-hermenéutico y teoría de representaciones sociales, que empleó como técnicas entrevistas a profundidad y análisis de contenido. Los hallazgos mostraron que la violencia para regular comportamiento, validación por celotipia, medidas conciliatorias y el supuesto agrado de las mujeres son formas legitimadoras de violencia en parejas heterosexuales. Se concluye que esta violencia se ha legitimado y naturalizado por el anclaje de representaciones, estereotipos y creencias, que ratifican el control de los hombres sobre las mujeres, en especial de su sexualidad y su cuerpo, como cosas de propiedad para el deleite y formas de autoridad, poder, control y desigualdad, que, a su vez, son permeadas por el modelo patriarcal.

Palabras clave: Legitimación; Violencia de Pareja; Estructura de Género; Práctica Social. 
This study aimed to show the social representations related to female victims, aggressors and services providers as great legitimizers of violence against women, considering their gender and social practice in Barranquilla, Colombia. This is a qualitative research, with a phenomenologicalhermeneutic approach and the theory of social representations, which used in depth interviews and content analysis as data collection technique. Findings suggest violence used to regulate behavior, passionate jealousy, conciliatory strategies and the assumed women satisfaction are legal means of violence in heterosexual couples. We conclude this type of violence has been established due to social representations, stereotypes and beliefs that reinforce male dominance, especially over female sexuality and body as properties for their pleasure and forms of authority, power, control and inequality, which are imposed by the patriarchal model.

Keywords: Legitimizers; Partner Violence; Gender Structure; Social Practice.
El concepto de legitimación se aplica en áreas del Derecho, la Política, la familia; pero como categoría de análisis de la violencia ha cobrado importancia para las Ciencias Sociales. Hoy desde la teoría de legitimidad se intenta explicar teorías de la estabilidad política y justicia distributiva y teorías de poder y de autoridad (Foucault, 2005), a partir de las cuales se establece una obligación moral de obediencia a un determinado sistema de poder, que se reproduce generando impactos negativos en lo micro de la sociedad (Bourdieu, 200o).

Aquí resulta importante una aproximación al concepto. El vocablo "legitimación” proviene del latín legitimus, que hace referencia a hechos creados desde las leyes cuya aplicabilidad se enmarca dentro de lo legal, equitativo y no reprochable. También, equivale a justificación o habilitación. Este concepto se ha utilizado desde las Ciencias Sociales en el análisis de categorías sobre el poder, autoridad, relaciones sujetos-Estado, movimientos de protesta, violencia política y violencia de género (Barreto et al., 2009; Bourdieu, 2000; De Beauvoir, 2007; Foucault, 2005; Friedan, 2009).

En este contexto, la legitimación de la violencia en las relaciones de pareja se refiere al conjunto de normas creadas y aceptadas culturalmente e internalizadas mediante los procesos de socialización interinstitucional, facilitando con ello la utilización de la violencia como forma válida para resolver los conflictos, que puede ser explicada desde la teoría de las representaciones sociales dado que expone como incorporamos y orientamos nuestras ideas y prácticas a partir de conocimientos colectivos. Las representaciones sociales constituyen un conocimiento socialmente construido; mediante la conversación y de los medios de comunicación, los actores sociales llegan a consensos, crean y recrean estos conocimientos estructurados en representaciones sociales, las cuales tienen sentido en el contexto sociocultural de la sociedad a partir del cual se da el anclaje o sentido a la estructura de la representación social misma, lo que nos hace familiarizar y naturalizar el contenido de la representación (Navarro; Restrepo, 2013). 
Paralelamente Moscovici (2006 apud Acosta, 2006) concibe las representaciones sociales como una teoría de cambio social, pues es a través de la recreación de las mismas representaciones que vamos provocando recreación en las prácticas sociales. De aquí que, como lo plantea Martínez, Quintal y Amarís (2019), la concepción de la violencia como instrumento para resolver conflictos en las relaciones de pareja entre los géneros se hace legitima desde el entorno sociocultural.

Además, los conflictos creadores de confrontaciones violentas están acompañados de discursos sociales generadores de creencias que disponen y conservan a grupos y determinados sectores de la sociedad para realizar y legitimar acciones penales como asesinatos y genocidios. En ese sentido, la teoría feminista, en sus auscultaciones, da luces dentro de lo que ha sido heterodesignante a la mujer y que, a su vez, en una relación de ida y vuelta, conminó a la autoasignación femenina; todo validado desde el patriarcado que se ha extendido en todas las esferas de vínculo social entre hombres y mujeres; pero que aún no ha sido posible desvincular el dispositivo entre géneros para que cese la violencia entre parejas y exista una verdadera autodeterminación tanto femenina como masculina en la mediación de los afectos (Amorós, 1985; Amorós; Cobo, 2006; Amorós; De Miguel, 2007a; Barreto et al., 2009). En tal sentido, la legitimación a la que se refiere este estudio es la que a través de los procesos de poder mencionados se instala en las relaciones de pareja, allí donde se reproduce desigualdades entre géneros ingresando la violencia como enclave natural. Por ello, los estudios y el enfoque de la legitimidad es un coadyuvante para que, en las prácticas de atención desde lo público, puedan servir de soporte para diluir lo que tradicionalmente se ha hecho con estas comprensiones quedando en desventaja la mujer.

De tal forma, las creencias son justificadas y asumidas humanamente como comportamientos inevitables internalizándose a nivel individual en las relaciones cotidianas de pareja y en el ámbito social, lo cual posteriormente se naturaliza (Galtung, 1990 apud Martínez; Amar, 2017). Esta se correlaciona con los postulados de Bourdieu (2008) en que toda legitimación de la violencia tiene un carácter simbólico que logrando imponerse dentro de las relaciones disimula el carácter de fuerza que se funda en su propia fuerza y, a su vez, añade su propia fuerza. Es por ello que puede darse por parte de quien ejerce la violencia minimización de la culpa y vergüenza desde dos dimensiones: interna y externa.

La primera se precisa por una dialéctica semántica definida por el lenguaje que dentro de las relaciones ejerce una justificación moral como etiquetación eufemística y también comparación ventajosa, respectivamente. En este mecanismo, el proceder nocivo es asumido como un propósito loable y moralmente ético, convirtiendo la conducta censurable en un heroísmo. Aunado, eufemismos que encubren las acciones negativas que hacen uso de expresiones minimizadoras de esta acción y que, finalmente, por medio de lo comparativo que relaciona una acción negativa con otra de mayor gravedad entrega finalmente una evaluación de esta acción como no tan grave, considerándolas como no violentas (Bandura, 1999, 2002).

En cuanto a la segunda dimensión, se subdivide en dos subdimensiones: transaccional y censura. La primera es definida por la transferencia y difusión de la responsabilidad, a su vez como una distorsión de las consecuencias; los primeros dos mecanismos disminuyen la culpa del agresor frente a su comportamiento negativo y también distribuye la culpabilización entre todos los miembros del grupo, lo que reduce, así, la preocupación por la víctima y la responsabilización personal del daño producido. Para finalmente distorsionar las consecuencias, esto sucede particularmente cuando actúan individualmente y no pueden culpar a los demás de su acción negativa o evadir la responsabilidad personal (Bandura, 1991, 1999). Por ello, causar daño a otras personas es más fácil cuando se invisibiliza el sufrimiento y las consecuencias de este por quien lo produce.

Así mismo, dentro de lo externo, se cierra con una etapa de censura y tiene que ver con una forma de culpabilización y deshumanización. Aquí se minimiza la falta personal, culpabiliza la víctima de haber desencadenado el comportamiento dañino (Bandura, 1990), el cual no es una decisión del sujeto que lo comete, sino una respuesta ante la provocación. Por lo tanto, las mujeres víctimas son 
las responsables de las agresiones que reciben por parte del agresor; lo que conlleva deshumanizarlas teniendo que ver con las posiciones que son asumidas por el agresor, tanto frente a la recepción de la violencia como el sufrimiento de las víctimas. Esta acción produce distanciamiento y acercamiento. Con respecto a la primera se presenta por negación, naturalización, estereotipia, culpabilización de las víctimas, incomprensión y deshumanización, lo que implica asumir una actitud de aceptación y normalización en la que le es indiferente la situación del otro. Esta estrategia de no responsabilidad reduce la posibilidad de ocuparse del fenómeno de violencia (Fernández; Revilla, 2016).

Por el contrario, el acercamiento genera sentimientos de empatía y humanización frente al sufrimiento de la víctima. Y al humanizarlo permite la implicación y responsabilización. La deshumanización es el mecanismo más perverso, por el impacto negativo que genera tanto para las víctimas como para las sociedades que hacen uso de este, debido a que se les despoja de su condición humana como sujetas de derechos, convirtiéndolas en objeto y, aún más grave, como un ser indigno de todo derecho y no merecedor de respeto. Al atribuirle estas cualidades de perversidad, se hace más fácil dañarlo (Bandura, 1999, 2002).

Finalmente, toda legitimación en las relaciones de pareja hace parte de lo intangible en sus afectos; aquello que no se ve y que puede estar internalizado en hombres y mujeres, ya sea por aprendizajes intergeneracionales, o por lo natural, en torno a la desventaja femenina y que se valida en las dos vías, de quien la ejerce y de quien es ejercida. A su vez, las políticas públicas no han podido actuar con todo el peso legal para que se pueda minimizar y erradicar este fenómeno, que no solo trae consigo detracción psicológica y física, sino que a raíz de los feminicidios es lo más cruel para las mujeres.

\section{Legislación colombiana contra la violencia de género}

En Colombia, hay importantes avances legislativos dirigidos a asegurar a las mujeres el ejercicio de derechos humanos en general y del derecho a una vida libre de violencia en particular. Para el tema de violencia sexual en adultos, niños/as y adolescentes, se crearon las leyes 360/1997 y 1146/2007 (Derechos..., c2020). La Ley 1.542/2012 contribuye a establecer límites a la violencia doméstica e inasistencia alimentaria, y la Ley 1.719/2014 plantea medidas para garantizar el acceso a la justicia a las víctimas de abuso sexual, sobre todo en el conflicto armado (Colombia, 2012, 2014).

Desde 2008 las leyes se han enfocado en la tipificación de la violencia física, sexual, psicológica, económica y patrimonial y en la reglamentación de la discriminación, los feminicidios y ataque por ácidos; para ello se fundamentan en la Ley 1257/2008, la Ley 1761/2015 y la Ley 1773/2016. Estas dos últimas tuvieron denominación de dos mujeres Rosa Elvira Celis y Natalia Ponce que fueron víctimas una de feminicidio y otra del ataque con ácido en su rosto. Finalmente, desde 2006, existe el Observatorio de Asuntos de Género mediante la Ley 1009/2006 y la Consejería Presidencial para la Equidad de la Mujer (Colombia, 2020), que tuvo inicio en 1999 y se ha fortalecido en 2019.

En el departamento del Atlántico, distrito de Barranquilla donde está ubicado este estudio; hay avances con la creación de la Mesa Interinstitucional para erradicar la violencia contra las mujeres cuya base legal es el Decreto n. ${ }^{\circ}$ ooogo7 de 2015, modificado parcialmente por el Decreto n. ${ }^{\circ}$ ooo298 de 2017 (Mesa..., 2018). De acuerdo al seguimiento de esta mesa en 2016, se realizó un estudio cuyos resultados permitieron identificar los alcances y desafíos de la Ley 1257 de 2008 en las instancias legales de las rutas de atención; en las cuales aún no se garantiza la atención integral a las mujeres víctimas de violencia de género (López; Orozco; Altamar, 2016). En suma, gracias a la unión de la sociedad civil, académicos, con el nivel político en Colombia, se avanza en la incorporación del establecimiento de una ruta eficaz para la comprensión fenomenológica de esta realidad que vivencian muchas parejas y que afecta principalmente a las mujeres; porque la violencia es el primer desencadenamiento para un feminicidio considerado en la actualidad como un salvajismo, lo cual conlleva señalar que la persistencia de este problema no se debe a la falta de mecanismos normativos, sino a la deficiencias en la aplicación de la protección integral 
y al desconocimiento de concepciones, creencias, estereotipos y representaciones sociales que tienen mujeres víctimas, hombres agresores y prestatarios/as de servicios sobre la violencia de pareja. Es por ello que el estudio se planteó como pregunta principal, ¿qué representaciones sociales de mujeres víctimas, hombres agresores y prestatarios/as de servicios legitiman la violencia en las relaciones de pareja desde la estructura de género como práctica social?

\section{Legitimación de la violencia de pareja desde el patriarcado}

En medio de la Revolución Francesa en 1791, las mujeres, por primera vez, alzaron sus voces desafiando el patriarcado. Olympe de Gouges propuso que análogo a los derechos del ciudadano se instalaran los derechos de la ciudadanía. La incomprensión del momento hizo que años más tarde la guillotinara por semejante propuesta; de ahí en el trascurso de estos siglos, ha aparecido un movimiento activo y teórico en que las feministas y la construcción de la teoría han venido aportando al desarrollo de la mujer en el mundo.

Amorós (1985), no obstante, plantea que el patriarcado que encierra la desventaja femenina desde niveles macros a micros, públicos y privados, mantiene aún su dominación, que finalmente es la interconexión de las instituciones y la microfísica del poder dentro de la familia, la iglesia, la escuela; que intergeneracionalmente se replica y hace que prevalezca en las distintas esferas sociales del planeta (Bourdieu, 200o; Foucault, 2005). Es así como se sabe que las mujeres en la edad antigua eran confinadas al gineceo, un lugar solo para ellas, donde había la dedicación contemplativa al mundo privado y doméstico; así la subordinación se concretaba en el encierro de las mujeres cautivas y cautivadas en el mundo patriarcal cuyo eje central de este cautiverio es la opresión de ellas. Para la mayoría de las mujeres, la vivencia del cautiverio significa sufrimiento, conflictos, contrariedades y dolor, para otras simboliza felicidad. Por lo tanto, todas las mujeres se encuentran cautivas en el mundo patriarcal por el solo hecho de ser mujeres.

Sin embargo, desde autoasignaciones femeninas, se abre un nuevo espectro para las mujeres construido por su misma comprensión y consciencia del rezago histórico del liderazgo femenino en los logros instalados a partir de sus derechos civiles, políticos y económicos (Amorós, 1985; De Beauvoir, 2007; Friedan, 2009; Lagarde, 2005; Lamas, 1986). Entre los caminos que han conducido al cautiverio de las mujeres, Lagarde (2005) expone la expropiación sexual del cuerpo, de bienes materiales y simbólicos de las mujeres y de su capacidad para contribuir al ordenamiento del mundo. Desde esta óptica, la subjetividad de las mujeres se construye a partir de la dependencia y del ser del otro. Sin embargo, autoras como De Beauvoir (2007) y Friedan (2009), además de revelarse contra el patriarcado, también hacen análisis de lo intrínseco a la mujer de su propia subjetividad y una reflexión del mundo privado (doméstico), respectivamente.

De esta manera, la visión patriarcal de la vida de las mujeres, instalada en la dominación, integra la violencia en las relaciones de pareja heterosexuales, que se refiere a los comportamientos orientados a producir daño a otra persona, en los que una parte vulnera los derechos humanos de la otra parte. Esta violencia impacta negativamente la salud de las mujeres que la padecen, así como su contexto familiar y socioeconómico.

La Organización Mundial de la Salud (OMS, 2016) advierte los efectos que esta violencia tiene sobre las mujeres cuando afirma que una de cada tres mujeres en el mundo ha sufrido violencia física y sexual por su pareja. En Colombia se incrementa la posibilidad de ocurrencia, contribuyendo con su normalización y legitimación, por medio de los siguientes factores: el género como una de las estructuras de práctica social, las normas sobre masculinidad, las inequidades económicas y las conductas relacionadas con el consumo de sustancias psicoactivas y alcohol (Derechos..., c2020). Por ello, se justifican culturalmente creencias, mitos y patrones de conducta de los individuos. Finalmente, muchas mujeres en la contemporaneidad, con toda la apertura legal, educativa y teórica, aún asumen el sentido patriarcal en sus vidas. Develar representaciones sociales de mujeres víctimas, agresores y prestatarios/ as de servicios legitimadoras de violencia de pareja, desde la estructura de género y práctica social en el distrito de Barranquilla, se constituye en un imperativo ético para las Ciencias Sociales. 


\section{El género como una de las estructuras de la práctica social y organización de la masculinidad}

El género como categoría de análisis se empezó a utilizar para referirse a la construcción sociocultural de los comportamientos, actitudes y sentimientos de hombres y mujeres. Lo que establece con su significado es que estas diferencias no son naturales y estas asociaciones no proceden de la naturaleza biológica de cada sexo, sino que son una construcción social, afianzada por las prácticas sociales (Oliveira, 2014). Butler (2007) y Connell (2015) coinciden con Bourdieu (1998 apud Oliveira, 2014) al vincular el concepto de género a la cultura, historia y relaciones de poder. Sin embargo, aunque los significados varían con cada cultura, un sistema de sexo-género está íntimamente ligado con factores políticos y económicos en cada sociedad; por ello es un sistema simbólico que coloca en tensión la relación de sexo con determinados contenidos culturales, según los valores y jerarquías sociales (Amorós; De Miguel, 2007b; De Lauretis, 2000). De esa manera, Connell (2015) expone cuatro dimensiones-hegemonía, subordinación, complicidad y marginación - que han permitido la estructuración del género desde las relaciones sociales resaltando su prevalencia.

En conclusión, desde estas concepciones sobre género se han construido prejuicios y creencias que afectan las relaciones de pareja y ponen en riesgo el derecho a una vida sin violencia por parte de las mujeres, debido al establecimiento de relaciones marcadas por la desigualdad, subordinación y estereotipos, puesto que "su principal finalidad es de sometimiento y control de la víctima por parte del agresor por medios coercitivos, generadores de miedo, inseguridad, sufrimiento y dolor" (Orozco, 2017, p. 265). De ahí que el desafío apunte a develar las formas de legitimación y reproducción, que desde las propias mujeres marquen su libertad y desarrollo de sus potencialidades en la vida pública y privada.

\section{Metodología}

El enfoque que orientó el estudio fue el cualitativo, con diseño fenomenológico-hermenéutico. El método de este diseño permite mediante la reflexión captar lo esencial del fenómeno que comprender, en este caso las representaciones sociales que legitiman la violencia en parejas heterosexuales desde el género como una de las estructuras de práctica social, a partir de los participantes en el fenómeno mismo. De forma que al encontrar las diferencias y las uniformidades de las descripciones otorgadas al fenómeno, finalmente se pueda dar cuenta de la estructura general de lo comprendido al respecto. Análisis que, conforme señala Giorgi, Giorgi y Morley (2017), no es forzado, sino que se sustenta en el ir y venir sobre las unidades consentidas en una reflexión constante que lleva a identificar los significados captados en todo el proceso.

En el estudio participaron doce mujeres víctimas de violencia de pareja, tres hombres agresores y ocho prestatarios/as de servicios, ubicados en Barranquilla. En total sumaron 23 participantes. La elección de esta población para la entrevista obedece al objeto principal del estudio de que son las representaciones sociales de la violencia de pareja que tienen las propias mujeres víctimas, hombres agresores y prestatarios/as de servicio, que permita develar su complejidad, legitimación y aspectos que la visibilizan. Su selección fue por medio del muestreo en cadena o bola de nieve y aceptación voluntaria, que consiste en "encontrar un caso perteneciente al grupo objeto de investigación y éste lleva al siguiente y al próximo y así sucesivamente hasta alcanzar el nivel de información suficiente para dar por terminada la investigación" (Patton, 1988 apud Sandoval, 1996, p. 123).

Los criterios de inclusión del estudio fueron: mujeres mayores de 18 años que interpusieron denuncia a causa de violencia en instituciones que integran la ruta; hombres mayores de 18 años, judicializados por el delito de violencia de pareja y funcionarios/as mayores de 18 años vinculados/as a instituciones estatales de los cuatro sectores: sector justicia -Fiscalía General de la Nación, Instituto Nacional de Medicina Legal y Ciencias Forenses y Comisarías de Familia, Oficina de la Mujer-; sector salud -Secretaría de Salud Distrital-; sector educativo -Secretaría de Educación Distrital-; sector Ministerio público -Defensoría del Pueblo. Como técnica se utilizó la entrevista a profundidad 
con previo consentimiento de los participantes, y el instrumento fue validado por dos expertas en el tema de violencia de género, y prueba piloto (Tabla 1). Los temas cubiertos en las entrevistas fueron: masculinidad como dominación y eje de las desigualdades de género; el sentido de propiedad masculina sobre el cuerpo de la mujer y conservación del honor masculino, las violencias sutiles e invisibles contra las mujeres en las relaciones de pareja; justificación de la violencia de pareja por el consumo de sustancias psicoactivas y alcohol; y discursos vs. prácticas legitimadoras de violencia de pareja.

\section{Tabla I - Preguntas orientadoras de la entrevista}

\begin{tabular}{|c|c|c|c|c|}
\hline Categoría & Subcategorías & $\begin{array}{l}\text { Guía de entrevista } \\
\text { mujeres víctimas }\end{array}$ & $\begin{array}{l}\text { Guía de entrevista } \\
\text { hombres agresores }\end{array}$ & $\begin{array}{l}\text { Guia de entrevista } \\
\text { prestatarios/as de } \\
\text { servicios }\end{array}$ \\
\hline \multirow{4}{*}{$\begin{array}{l}\text { Legitimación } \\
\text { social de la } \\
\text { violencia como } \\
\text { castigo hacia } \\
\text { la mujer }\end{array}$} & $\begin{array}{l}\text { Legitimación } \\
\text { de la violencia } \\
\text { para regular el } \\
\text { comportamiento }\end{array}$ & $\begin{array}{l}\text { I. ¿Culturalmente la mujer } \\
\text { dentro de una relación de } \\
\text { pareja ha sido asumida como } \\
\text { la propiedad del marido, por } \\
\text { tanto, esto le daría derecho a } \\
\text { agredirla para disciplinarla? } \\
\text { 2. ¿Las mujeres con su } \\
\text { cantaleta terminan por } \\
\text { provocar la agresión? }\end{array}$ & $\begin{array}{l}\text { I. ¿Muchos hombres ejercen } \\
\text { violencia cuando sienten que } \\
\text { han perdido el control sobre } \\
\text { algún aspecto de su vida? } \\
\text { 2. ¿Todos los hombres están } \\
\text { predispuestos a ser violentos? } \\
\text { 3. ¿Las mujeres con su } \\
\text { cantaleta terminan por } \\
\text { provocar la agresión? }\end{array}$ & $\begin{array}{l}\text { I. ¿Culturalmente la mujer } \\
\text { dentro de una relación de } \\
\text { pareja ha sido asumida } \\
\text { como la propiedad del } \\
\text { marido, por tanto, esto le } \\
\text { daría derecho a agredirla } \\
\text { para disciplinarla? } \\
\text { 2. ¿Las mujeres con su } \\
\text { cantaleta terminan por } \\
\text { provocar la agresión? }\end{array}$ \\
\hline & $\begin{array}{l}\text { Validación por } \\
\text { celotipia }\end{array}$ & $\begin{array}{l}\text { 3. ¿Considera que los celos } \\
\text { son un motivo para que su } \\
\text { pareja ejerza violencia? } \\
\text { 4. ¿Las instituciones } \\
\text { deberían atender la } \\
\text { problemática de los celos en } \\
\text { las relaciones de pareja? } \\
\text { 5. ¿Qué estrategias utilizan } \\
\text { los prestatarios/as de } \\
\text { servicios para su orientación, } \\
\text { resolución y manejo frente a } \\
\text { la denuncia por celos? }\end{array}$ & $\begin{array}{l}\text { 4. ¿Considera que los } \\
\text { celos son un motivo para } \\
\text { ejercer violencia? } \\
\text { 5. ¿Las mujeres celosas se } \\
\text { buscan la agresión por parte } \\
\text { del hombre? } \\
\text { 6. ¿Las instituciones } \\
\text { deberían atender la } \\
\text { problemática de los celos en } \\
\text { las relaciones de pareja? } \\
7 \text { · ¿Qué estrategias utilizan } \\
\text { las instituciones para su } \\
\text { orientación, resolución } \\
\text { y manejo? }\end{array}$ & $\begin{array}{l}\text { 3. ¿Las instituciones } \\
\text { deberían atender la } \\
\text { problemática de los celos en } \\
\text { las relaciones de pareja? } \\
\text { 4. ¿Qué estrategias utilizan } \\
\text { para su orientación, } \\
\text { resolución y manejo? }\end{array}$ \\
\hline & $\begin{array}{l}\text { Continuación } \\
\text { de la relación } \\
\text { mediante la } \\
\text { conciliación }\end{array}$ & $\begin{array}{l}\text { 6. Cuando usted hace la } \\
\text { denuncia, ¿qué es más } \\
\text { viable en los casos de } \\
\text { violencia de pareja, la } \\
\text { separación o la continuidad? }\end{array}$ & $\begin{array}{l}\text { 8. En relación con su } \\
\text { experiencia, ¿qué es más } \\
\text { viable en los casos de } \\
\text { violencia de pareja, la } \\
\text { separación o la continuidad? }\end{array}$ & $\begin{array}{l}5 \text {.En relación con su } \\
\text { experiencia, ¿qué es } \\
\text { más viable en los casos } \\
\text { de violencia de pareja, } \\
\text { la separación o la } \\
\text { continuidad? }\end{array}$ \\
\hline & $\begin{array}{l}\text { Legitimación por } \\
\text { supuesto agrado } \\
\text { de las mujeres }\end{array}$ & $\begin{array}{l}\text { 7. ¿Aunque su pareja la } \\
\text { agreda está dispuesta a } \\
\text { tolerar porque no sabe } \\
\text { vivir sin ella, y tampoco se } \\
\text { atreve a dejarlo? } \\
\text { 2. ¿Considera usted que hay } \\
\text { mujeres que les gusta que } \\
\text { las agredan? }\end{array}$ & $\begin{array}{l}\text { 9. ¿Considera usted que hay } \\
\text { mujeres que les gusta que } \\
\text { las agredan? } \\
\text { 10. ¿Las mujeres que se visten } \\
\text { de manera provocativa se } \\
\text { buscan la agresión? }\end{array}$ & $\begin{array}{l}\text { 6. ¿Considera usted que hay } \\
\text { mujeres que les gusta que } \\
\text { las agredan? }\end{array}$ \\
\hline
\end{tabular}


A partir del análisis de contenido, se realizó la interpretación de resultados y codificación, "por ésta se detectan y señalan los elementos relevantes del discurso verbal o no verbal, y a su vez éstos se agrupan en categorías de análisis" (Álvarez-Gayou, 2003, p. 164), mediante el siguiente procedimiento: (1) Identificación de unidades de análisis; (2) Clasificación de unidades de análisis por categorías y subcategorías; (3) Comparación de categorías entre sí para agruparlas en temas y buscar posibles vinculaciones; (4) Análisis descriptivo individual; (5) Análisis interpretativo; (6) Triangulación de los relatos proporcionados por los participantes; (7) Identificación de representaciones legitimadoras de violencia en las relaciones de pareja. A continuación, se presentan resultados de una de las 11 categorías y subcategorías que emergieron de la subjetivación desde las voces de mujeres víctimas, hombres agresores y prestatarios/as de servicio.

\section{Resultados y discusión}

En este esfuerzo hermenéutico-fenomenológico por comprender las epistemologías y significados de la violencia en las relaciones de pareja ancladas en el modelo patriarcal, es importante conocer las formas en las que se continúa legitimando estas violencias en ámbitos privados y públicos. Se constata la transversalidad en la constitución de la dominación como un máximo, aunque es más una dialéctica instaurada por los varones y a su vez validada por las mujeres, y la incidencia de la práctica cultural. En este sentido, el orden de las cosas se presenta a un tiempo en su estado objetivo -tanto en las cosas como en el mundo social-; en los cuerpos y en los hábitos de sus agentes, que funcionan como sistemas de percepciones, en el pensamiento y acción (Bourdieu, 200o) que, a su vez, derivan una interconexión de las instituciones en sus estructuras de poder (Foucault, 2005). Esto impide el reconocimiento de los derechos de las mujeres cuando, desde la familia, las instituciones del Estado y las prácticas culturales coartan a la mujer dentro de sus libertades, lo que hace que en muchos casos valide la situación y reincida en la conciliación, y no la hace tener un juicio valorativo de amor propio.
Así mismo, dentro de las microfísicas de la cotidianidad como en las relaciones de pareja hay incidencia de los hombres en la forma de vestir, en la manera de comportarse, en la elección de sus amistades y en las actividades recreativas como principales estrategias para ejercer dominio y control. Frente a estas evidencias, el cuerpo femenino es controlado y regulado por medio de discursos y prácticas debido al régimen disciplinario de la feminidad. De esta manera, Foucault (2005) le denomina anatomía política; es decir, la física del poder, el dominio sobre el cuerpo, se efectúa de acuerdo con las leyes de la óptica y de la mecánica bajo todo un juego de espacios, de pantallas y sin recurrir al exceso, a la fuerza, a la violencia (Foucault, 2005).

A la luz de la anterior comprensión, el cuerpo de las mujeres puede ser disciplinado, sometido, transformado y perfeccionado desde la era clásica hasta el día de hoy. Así lo evidenció la categoría legitimación social de la violencia como castigo hacia la mujer y subcategorías emergentes en relatos de mujeres víctimas y hombres agresores como la legitimación de la violencia para regular el comportamiento y validación por celotipia, esta última también presente en prestatarios/as de servicios. Otras como continuación de la relación mediante la conciliación y legitimación por supuesto agrado de las mujeres resultaron del análisis de relatos de mujeres víctimas y prestatarios/as de servicio. Estas conducirán a develar las prácticas y representaciones de la masculinidad hegemónica, los códigos de construcción cultural y su relación entre violencia de pareja y masculinidad. En relación a la primera subcategoría, los relatos de mujeres evidencian resistencia frente a esta representación. Uno de los testimonios así lo confirmó: "Por eso fueron los problemas que tuvimos siempre, porque él quiso disciplinarme, tomarme como si yo fuera de él, pero yo no soy de él, tengo mi propia identidad, mi propia autonomía” (U. de A. 10o, F, E. 28 años). Este relato demuestra el control ejercido por medio de la violencia, así como ideologías que obligaban a las mujeres a permanecer en el espacio privado. Es a partir de estas prácticas que en los años 70 surge el concepto de patriarcado como formas de dominación 
mediante el género y modelo dominante de la raza humana. Esta idea comprendía muy bien el poder y la misoginia de una estructura de relaciones sociales conformada por el Estado, la economía, la cultura, las comunicaciones, el parentesco, la educación y la sexualidad (Connell, 2015). Esas estructuras se instauran y reconstituyen por la práctica, porque esta no actúa en el vacío, sino forma al mundo, la realidad en la cual nos desenvolvemos y vivimos.

Las prácticas de dominación y control legitimadas por los saberes hegemónicos de las diferentes épocas una vez normalizadas en el cuerpo y subjetividades de los varones los predisponen a permanecer en la esfera de dominación (Sambade, 2015), lo que se hizo evidente en el relato de agresores al reconocer prácticas de violencia por la incapacidad de gestionar sus emociones: "Sí, porque perdemos el control, porque hay cosas que no nos quieren salir bien" (U. de A. 27, M. E. 46 años). Lo que explicaría Connell (2015) como la interconexión de la masculinidad, el poder y la violencia en la historia de la humanidad. En sus testimonios se interpreta la evasión de su culpa, justificación de sus acciones violentas y el afán de hacer comprensibles sus acciones ante sus interlocutores: "Todo eso que ella me hacía lo acumulé, lo que pasó fue un impulso, yo no soy así, creo yo que es un problema psicológico por lo que he estado viviendo y aguantando" (U. de A. 272, M. E. 36 años).

En este relato el hombre aparentemente no es violento, la situación fue lo que desencadenó la violencia. De esta manera, minimizan la falta personal culpabilizando a la víctima de haber desencadenado el comportamiento violento; la gravedad de la internalización de este mecanismo por parte del sujeto agresor radica en la pérdida de todo sentimiento de culpa y reforzamiento de sentimientos de complacencia, lo que conlleva la legitimación para el incremento y escalada de violencia (Bandura, 1991). Además, asocian a creencias de que las mujeres con su cantaleta terminan por provocar la agresión: "Entonces yo siento que sí, que a veces uno puede provocar con las palabras que ellos lleguen a otros extremos" (U. de A. 139, F, edad 32 años). Estas creencias tienen su fundamento en el autoengaño, hasta el punto de naturalizarlas, obligándolas a permanecer en los roles sexuales y sociales asignados del cuidado y reproducción. Lo que corresponde con Díez (2017) en la noción de creencia como disposición, es decir, la creencia conlleva la actuación con la convicción de que está haciendo lo correcto. En este sentido, cuando los sistemas de creencias resultan ser resistentes al cambio en contextos de violencia de pareja cumplen una función justificadora y legitimadora de la violencia en vez de aminorar su aparición (Galtung, 1990 apud Martínez; Amar, 2017)

También se legitima por celotipia al ser considerada por participantes del estudio como el principal motivo para ejercer violencia. Escuchemos la voz de un agresor: "Los celos son peligrosos, porque te cogen la mente y te la transforma en un momentico y haces cosas malas con tu pareja” (U. de A. 295 M. E. 36 años). Igualmente prestatarios/as de servicios ubicaron los celos entre las principales causas de denuncia y motivos de agresiones hacia las mujeres por parejas y exparejas: "En la mayor parte de los casos atendidos, los celos en la pareja de parte del hombre hacia a la mujer, son los causantes de la violencia física, psicológica, económica y sexual" (Secretaría de la Mujer, U. de A. 414, F. E. 28 años).

En relación con las mujeres víctimas, sus relatos están impregnados de violencia por celos: "Él me celaba con todo el mundo y era uno de los motivos por los cuales me violentaba con frecuencia" (U. de A. 49, F. E, 45 años). Estos hechos, que según testimonios de hombres agresores fueron los causantes del deterioro de las relaciones y que a su vez validan sus comportamientos violentos, están acompañados de afirmaciones tales como "usted me entiende" (U. de A. 274, M. E. 46 años), "usted sabe que es así" (U. de A. 24O, M. E, 46 años), lo que permite identificar al interior de sus relatos la existencia de códigos culturales relacionados con la violencia de pareja y masculinidades hegemónicas.

Uno de esos códigos culturales es el rol de las emociones subjetivas en los hechos violentos percibidos por los agresores como normales entre los motivos generadores de las acciones violentas. Al mismo tiempo de ser atribuciones subjetivas, están interconectadas con demarcaciones sociales y simbólicas utilizadas dentro de las jerarquías de género descritas por Jimeno (2007 apud Quiroz; Pineda, 2009, p. 100) como "sentimientos subjetivos, modelos culturales sobre el amor, la pareja, 
la fidelidad, la fuerza masculina, la emoción y la razón en el sujeto humano, se vuelven medios de subordinación o dominación social de las mujeres frente a los hombres". Pero por ser este un aprendizaje social puede ser modificado y cambiado, siempre y cuando los agentes socializantes como la familia escuela, iglesia, Estado y medios de comunicación estén dispuestos a hacerlo (Connell, 2015)

La continuidad de la relación mediante la conciliación son medidas que, a pesar de no estar contempladas en la normatividad vigente, siguen siendo promovidas por algunas instituciones a fin de resolver los conflictos, constituyéndose esta práctica en una forma de vulneración del derecho a la justicia y un atentado contra la dignidad de las mujeres. Lo que se hizo evidente en el siguiente relato: "Me aconsejaban la continuidad, hacer una conciliación, primero ellos hacen una conciliación de pareja, tratan de hacer volver después que esa persona te partió, te hizo y deshizo, tratan de volver a unirnos, las decisiones son conciliatorias" (U. de A. 52, F. E. 28 años). De esta forma, se puede explicar la reincidencia de los casos de violencia en las relaciones de pareja. Entre los motivos que argumentaron las mujeres participantes en el estudio están: La vulneración de los derechos y desconocimiento de estos; la ausencia de tratamientos integrales; poco seguimiento a los casos y evolución de los mismos por parte de las instituciones responsables; el miedo a la denuncia y enfrentar la incertidumbre; la falta de autonomía económica de las mujeres; y los celos.

La legitimación por supuesto agrado de las mujeres es el mecanismo con mayor arraigo cultural legitimador de violencia de pareja, por hacer parte de los discursos de los tres actores: "Por lo menos hay mujeres que sí, que son masoquistas" (U. de A. 201, F. E. 28 años). Sin embargo, uno de los testimonios develó la dependencia emocional, más que la dependencia sexual y económica: "No es gustar, a nadie le gusta que lo maltraten, de pronto cuando uno está fuera de ese círculo, puedes decir que es masoquista y no es eso, es que se vuelve como una dependencia, es como un vicio, igual, es como un vicioso de droga, tú quieres salir de eso pero no puedes, porque hay una dependencia emocional, tanto para el agresor como para el agredido" (U. de A. 53,
F. E. 28 años). La interpretación de este relato es de gran utilidad para mostrar el anclaje de los ciclos de violencia en la cotidianidad de las relaciones de pareja, que a pesar de constituirse en un delito y vulneración de los derechos humanos de las mujeres continua presente en los estados de buenas conciencias y en la moral religiosa de las familias.

Al respecto la segunda medición sobre la tolerancia social e institucional de las violencias contra las mujeres enfatizó las consecuencias que estas representaciones tienen sobre la vida de las mujeres, resaltando las siguientes: Culpabilización a las víctimas por persistir en este tipo de relaciones; débil respuesta por parte de las instituciones estatales las cuales no ofrecen alternativas de solución de manera coordinada, ágil e integral; la garantía de la no repetición de las agresiones es responsabilidad de las mujeres, por la retractación después de colocar la denuncia y la justificación de la no intervención de terceros en problemas de pareja por el reconocido adagio "en peleas de marido y mujer nadie se debe meter" (Colombia, 2015, p. 65). El supuesto agrado invisibiliza y minimiza la violencia contra las mujeres en las relaciones de pareja e implica la legitimación por aparente falta de riesgo y peligro de la agresión para la vida de las mujeres, de ahí que esta se ha convertido en uno de los mecanismos para la solución de conflictos en la familia y la sociedad en general. Por lo tanto, romper con las relaciones asimétricas de poder que propician la condición de víctima, en todos los escenarios de la realidad, es hoy un imperativo ético, así como la generación de una cultura de paz.

Finalmente, esa transversalizacion del poder interconectado tiende a invisibilizar el sentir de la mujer en sus distintas formas violentas dentro de la familia y la sociedad, aunque hay caminos posibles y se abre un espectro institucional en torno al análisis de datos estadísticos y la formación de funcionarios (Bogotá, 2019; Cada..., 2019; Colombia, 2019; Comisarios..., 2015) para comprender lo complejo de la situación de las mujeres no solo en relación a la derivación de la violencia, sino de la desventaja histórica en la que se ha encontrado durante siglos. Por ello, es necesario que cada vez más las mujeres puedan autoasignarse tomando distancia de las heterodesignaciones patriarcales 
(Femenías, 2007) y ser, a su vez, un agente de su propio desarrollo (Sen, 2010) separado de la validación del otro, encontrándose con ella misma en sus perspectivas hacia su autorespeto que conlleven sus realizaciones plenas más allá de los roles que les corresponda afrontar.

\section{Conclusiones}

De acuerdo a este proceso de investigación, dentro de los principales hallazgos encontrados, hay alta valoración de los esfuerzos realizados en Colombia, en el departamento del Atlántico y, en particular, en el distrito de Barranquilla, tanto en la legislación como en la ratificación de instrumentos normativos en materia de protección de los derechos humanos de las mujeres. Sin embargo, en la práctica se ha identificado obstáculos en la aplicación de estas normas al momento de abordar el fenómeno de violencia contra las mujeres en las relaciones de pareja. Entre los principales obstáculos identificados en los relatos de participantes se resaltaron los siguientes: Incidencia de prejuicios y estereotipos arraigados en los prestatarios/as de servicios los cuales salen a relucir durante la atención; uso de medidas conciliatorias exponiendo a la mujer al peligro inminente de ser asesinada; legitimación de la violencia por celotipia y supuesto agrado de las mujeres. Los mencionados obstáculos se constituyen en los principales nudos críticos para el acceso a la justicia de las mujeres que acuden a las instituciones responsables de su atención y protección.

En tal sentido, el aporte de este estudio es importante a las Ciencias Sociales debido a que se construye desde las voces participantes, quienes han experimentado este fenómeno, del cual puede servir de soporte para fortalecer las rutas de atención para que los funcionarios desde todos los ámbitos puedan comprender cada situación particular sin el sesgo de género, lo que podría evitar los feminicidios en el que Barranquilla tiene un foco nacional, del cual es necesario confrontar esta situación desde la validación científica y legal. Del mismo modo, la reivindicación que los participantes experimentaron en las entrevistas es uno de las sustanciales contribuciones que se hace en el rol de investigadora; los actos de habla generaron un proceso mental importante, que sistematizados y analizados brindan representaciones ancladas en el fenómeno, pero que si son tenidas en cuenta desde la política pública, pueden dar luces para prevenir la violencia de pareja en Barranquilla. Así tanto hombres como mujeres puedan comprenderse sin entrar en un grado agudo de la relación violenta, que es uno de los pasos previos al feminicidio.

Lo anterior se aúna a la abstracción que produjo esta investigación desde aspectos contextuales, legales y empíricos, en el nivel internacional y local, en relación a la violencia de pareja; lo cual arrojó el conocimiento de los tipos de violencia, así como la violencia estructural producida y reproducida por las diferentes formas de exclusión, desigualdad y discriminación en las que están inmersas las mujeres. Esto devela una encrucijada femenina en la contemporaneidad que de no detenerse puede seguir reproduciéndose, limitando el bienestar y desarrollo pleno de la mujer en sus distintos roles.

Finalmente, se constata la naturalización de la legitimación e inobservancia por parte del Estado debido a la poca credibilidad en sus instituciones administradoras de justicia, el incumplimiento de la ley y normas internacionales ratificadas, pero aún más grave la impunidad, que es la forma más perversa de naturalización y legitimación institucional de la violencia contra las mujeres. En este sentido, la búsqueda del restablecimiento de derechos humanos de las mujeres se ve frustrada cuando no se le otorga por parte de prestatarios/as de servicios las medidas de atención, protección y administración de justicia de manera eficaz, además de la revictimización que exacerba el riesgo y devela los vacíos del sistema de justicia colombiano, que a su vez es permeado por el modelo patriarcal (ONU Mujeres, 2015).

\section{Referencias}

ACOSTA, M. La psicología de las minorías activas revisitada: entrevista con Serge Moscovici. Polis: Investigación y Análisis Sociopolítico y Psicosocial, Ciudad de México, v. 2, n. 1, p. 141-177, 2006.

ÁLVAREZ-GAYOU, J. L. Cómo hacer investigación cualitativa: fundamentos y metodología. Ciudad de México: Paidós, 2003. 
AMORÓs, C. Hacia una crítica de la razón patriarcal. Barcelona: Anthropos, 1985.

AMORÓS, C.; COBO, R. Interculturalidad, feminismo y educación. Madrid: Los Libros de la Catarata, 2006.

AMORÓS, C.; DE MIGUEL, A. Teoría feminista: de la ilustración a la globalización: de la ilustración al segundo sexo. 2. ed. Madrid: Top Printer Plus, 2007a. v. 1.

AMORÓS, C.; DE MIGUEL, A. Teoría feminista: de la ilustración a la globalización: de los debates del género al multiculturalismo. 2. ed. Madrid: Minerva, 2007b. v. 3.

BANDURA, A. Mechanisms of moral disengagement in terrorism. In: REICH, W. (Ed.). Origins of terrorism: psychologies, ideologies, theologies, states of mind. Cambridge: Cambridge University Press, 1990. p. 161-191. Disponível em: <https://bit.ly/3kbm5dN>. Acesso em: 11 abr. 2019.

BANDURA, A. Social cognitive theory of moral thought and action. In: KURTINES, W. M.; GEWIRTZ, J. L. (Ed.). Handbook of moral behavior and development. Hillsdale: Lawrence Erlbaum, 1991. v. 1. p. 45-103. Disponível em: <https://bit.ly/ 39ILgk8>. Acesso em: 11 abr. 2019.

BANDURA, A. Moral disengagement in the perpetration of inhumanities. Personality and Social Psychology Review, Thousand Oaks, v. 3, n. 3, p. 193-209, 1999. DOI: 10.1207/ s15327957pspro303-3

BANDURA, A. Selective moral disengagement in the exercise of moral agency. Journal of Moral Education, Abingdon, v. 31, n. 2, p. 101-119, 2002. DOI: $10.1080 / 0305724022014322$

BARRETO, I. et al. La legitimación como proceso en la violencia política, medios de comunicación y construcción de culturas de paz. Universitas Psychologica, Bogotá, DC, v. 8, n. 3, p. 737-748, 2009.

BOGOTÁ. Secretaria de Integracion Social. Las mujeres siguen siendo las que mas sufren violencia intrafamiliar. Bogotá, DC: Alcadía de Bogotá, 2019. Disponível em: <https://bit.ly/ 2ORMS31>. Acesso em: 14 jul. 2020.
BOURDIEU, P. La dominación masculina.

Barcelona: Anagrama, 2000.

BOURDIEU, P. La reproducción: elementos para una teoría de la enseñanza. Madrid: Editorial Popular, 2008.

BUTLER, J. El género en disputa: el feminismo y la subversión de la identidad. España: Paidós, 2007.

CADA hora se presentan denuncias por violencia doméstica y de género en Atlántico. El Heraldo, Barranquilla, 2 dez. 2019. Disponível em: <https://bit.ly/3pINkhI>. Acesso em: 2 fev. 2021.

COLOMBIA. Ley no. 1.257, de 4 de diciembre de 2008. Por la cual se dictan normas de sensibilización, prevención y sanción de formas de violencia y discriminación contra las mujeres, se reforman los Códigos Penal, de Procedimiento Penal, la Ley 294 de 1996 y se dictan otras disposiciones. Diario Oficial, Bogotá, DC, 4 dez. 2008.

COLOMBIA. Ley no. 1.542, de 5 de julio de 2012. Por la cual se reforma el artículo 74 de la Ley 906 de 2004, Codigo de Procedimiento Penal. Diario Oficial, Bogotá, DC, 5 jul. 2012. Disponível: <https://bit.ly/3jzL8GT>. Acesso em: 9 fev. 2021.

COLOMBIA. Ley no. 1.719, de 18 de junio de 2014. Por la cual se modifican algunos artículos de las leyes 599 de 2000, 906 de 2004 y se adoptan medidas para garantizar el acceso a la justicia de las víctimas de violencia sexual, en especial la violencia sexual con ocasión del conflicto armado, y se dictan otras disposiciones. Diario Oficial, Bogotá, DC, 18 jun. 2014. Disponível: <https://bit.ly/ 3a5QRRP>. Acesso em: 9 fev. 2021.

COLOMBIA. Presidencia de la República. Equidad de la Mujer. Segunda medición del estudio sobre tolerancia social e institucional de las violencias contra las mujeres. Bogotá, DC: Proyectamos Colombia, 2015.

COLOMBIA. Procuraduria General de la Nacion. Segunda vigilancia superior a las comisarías de familia. Bogotá, DC, 2019. Disponível em: <https://bit.ly/3tngYyg>. Acesso em: 14 jul. 2020.

COLOMBIA. Presidencia de la República. Consejería Presidencial para la Equidad de la Mujer. Reglamentación. Bogotá, DC, 2020. 
Disponível em: <https://bit.ly/3dCa8fZ>. Acesso em: 21 mar. 2020.

COMISARIOS de familia iniciarán maestría en estudios de género y violencia intrafamiliar. Gobernación del Atlántico, Barranquilla, 26 maio 2015. Disponível em: <https://bit.ly/30MlDmN〉. Acesso em: 14 jul. 2020.

CONNELL, R. Masculinidades. Ciudad de México: Universidad Nacional Autónoma de México, 2015.

DE BEAUVOIR, S. El segundo sexo. Bueno Aires: De Bolsillo, 2007.

DE LAURETIS, T. Diferencias: etapas de un camino a través del feminismo. Madrid: Horas y Horas, 2000.

DERECHOS de las víctimas de violencia de género. Ministerio de Salud de Colombia, Bogotá, DC, c2020. Disponível em: https://bit.ly/2YNqqKn>. Acesso em: 21 mar. 2020.

DÍEZ, P. A. Más sobre la interpretación (II): ideas y creencias. Revista de la Asociación Española de Neuropsiquiatría, Madrid, v. 37, n. 131, p. 127-143, 2017.

FEMENÍAS, M. El género del multiculturalismo. Buenos Aires: Quilmes, 2007.

FERNÁNDEZ, C.; REVILLA, J. Seres "humanos" o seres "lejanos": imágenes de violencia real e implicación/distanciamiento con las víctimas. Communication \& Society, Pamplona, v. 29, n. 3, p. 103-118, 2016.

FOUCAULT, M. Vigilar y castigar. Buenos Aires: Siglo XXI, 2005.

FRIEDAN, B. Mística de la feminidad. Madrid: Ediciones Catedra, 2009.

GIORGI, A.; GIORGI, B.; MORLEY, J. The descriptive phenomenological psychological method. In: WILLIG, C.; ROGERS, W. S. (Ed.). The Sage handbook of qualitative research in psychology. 2. ed. Thousand Oaks: Sage, 2017. p. 176-192. Disponível em: <https://bit. ly/2LiRRZ9>. Acesso em: 7 jul. 2020.

LAGARDE, M. Los cautiverios do las mujeres: madresposas, monjas, putas, presas y locas.
Ciudad de México: Universidad Nacional Autónoma, 2005.

LAMAS, M. La antropología feminista y la categoría de género. Revista Nueva Antropología, Ciudad de México, v. 8, n. 3o, p. 173-198, 1986.

LÓPEZ, E.; OROZCO, A.; ALTAMAR, L. La mudanza de sentidos: trazando límites para la erradicación de violencia contra de las mujeres desde una mesa interinstitucional. In: PATTARO AMARAL, F.; GONZÁLEZ MARTÍNEZ, M. N. (Org.). Deshilando las violencias de género y construyendo centros de resistencias. Barranquilla: Universidad Simón Bolívar, 2016. p. 183-225.

MARTÍNEZ, M.; AMAR, J. ¿Quien es el malo del paseo? Legitimación de la violencia por niños en contextos de migración forzada. Barranquilla: Universidad del Norte, 2017.

MARTÍNEZ, C.; QUINTAL, R.; AMARÍS, M. La violencia masculina en la pareja como proceso relacional: un desafío de superación cultural. Masculinities and Social Change, Barcelona, v. 8. n. 3, p. 307-331, 2019.

MESA departamental promueve acciones para erradicar violencia contra la Mujer. Gobernación del Atlántico, Barranquilla, 11 jun. 2018.

Disponível em: <https://bit.ly/2MLBcoM>. Acesso em: 21 mar. 2020.

NAVARRO, O.; RESTREPO, D. Representaciones sociales: perspectivas teóricas y metodológicas. CES Psicología, Medellín, v. 6, n. 1, p. i-iv, 2013.

OLIVEIRA, T. La maternidad como resistencia: el discurso del cuerpo. In: GONZÁLEZ, M. Género y discurso: fuerzas regulatorias dominantes y márgenes de getión de las autonomías. Barranquilla: Universidad Simón Bolívar, 2014. p. 87-139.

ONU MUJERES - ENTIDAD DE LAS NACIONES UNIDAS PARA LA IGUALDAD DE GÉNERO Y EL EMPODERAMIENTO DE LAS MUJERES. La misma historia otra vez: vivencias de mujeres y barreras de acceso a la justicia: Ley 1.257 de 2008. Bogotá, DC: Mesa por el derecho de las mujeres a una vida libre de violencias, 2015 . 
OMS - ORGANIZACIÓN MUNDIAL DE LA SALUD. Violencia contra la mujer. 2016. (nota descriptiva $\mathrm{n}^{0}$ 239). Disponível em: <https://bit.ly/3tDS8XU>. Acesso em: 27 mar. 2019.

OROZCO, A. La violencia de pareja y sus representaciones sociales: Distrito de Barranquilla, Colombia. In: AGUILAR, A.; OROZCO, A.; JIMÉNEZ M. (Comp.). Estudios doctorales femeninos: aportes desde las ciencias sociales y humanas. Barranquilla: Universidad Simón Bolívar, 2017. p. 249-277.
QUIROZ, F.; PINEDA, J. Subjetividad, identidad y violencia: masculinidades encrucijadas. Universitas Humanística, Bogotá, DC, n. 67, p. 81103, 2009.

SAMBADE, B. I. Sobre las contradicciones de la razón moderna y la constitución de la subjetividad. Prisma Social, Madrid, n. 13, p. 787-851, 2015.

SANDOVAL, C. Investigación cualitativa. Bogotá, DC: ICFES, 1996.

SEN, A. Desarrollo y libertad. Bogotá, DC:

Planeta, 2010.

\section{Contribución de los autores}

Orozco fue responsable de la concepción del estudio, análisis de los datos y redacción del artículo. Aguilar y Amarís ambas autoras contribuyeron con la fundamentación teórica y redacción del artículo.

Recibido: 26/03/2020

Re-presentado: 14/07/2020

Aprobado: 25/08/2020 